\title{
Comparative Docking Studies of Rosmarinic Acid and Sinesitin to Inhibit HSP70
}

\author{
Mansoureh Nazari $V^{1^{*}}$, Syed Mahmood ${ }^{2,3 *}$, Subashini Raman ${ }^{2}$ \\ ${ }^{1}$ Department of Pharmacology, School of Pharmaceutical Sciences, Universiti Sains Malaysia, Minden, 11800 \\ Pulau Pinang, Malaysia \\ ${ }^{2}$ Faculty of Engineering Technology, University Malaysia Pahang, Gambang, 26300 Malaysia. \\ ${ }^{3}$ Centre of Excellence for Advanced Research in Fluid Flow (CARIFF), University Malaysia \\ Pahang, Gambang, 26300, Malaysia. \\ *Email:nazarimansoure@gmail.com,syedmahmood@ump.edu.my
}

\begin{abstract}
The HSP70 family of heat shock proteins consists of molecular chaperones of approximately $70 \mathrm{kDa}$ in size that serve critical roles in protein homeostasis. These adenosine triphosphatases unfold misfolded or denatured proteins and can keep these proteins in an unfolded, folding-competent state. They also protect nascent translating proteins, promote the cellular or organellar transport of proteins, reduce proteotoxic protein aggregates and serve general housekeeping roles in maintaining protein homeostasis. The HSP70 family is the most conserved in evolution, and all eukaryotes contain multiple members. HSP70 family of proteins can be thought of as a potent buffering system for cellular stress, either from extrinsic (physiological, viral and environmental) or intrinsic (replicative or oncogenic) stimuli. Not surprisingly, cancer cells rely heavily on this buffering system for survival. The overwhelming majority of human tumors overexpress HSP70 family members, and expression of these proteins is typically a marker for poor prognosis. In this article the tendency of rosmarinic acid and sinesitin towards HSP70 were analyzed to predict anticancer effect of these compound by inhibiting this molecule. Moreover, ATP was selected as positive control.
\end{abstract}

Keywords: HSP70, Auto dock4.2, Cancer, ATP.

\section{INTRODUCTION}

Cancer is a lethal disease that requires complicate therapeutic strategies to overcome. The rate of human death yearly due to cancer is $57 \%$ of the cases worldwide [1].

The family of human HSP70 consists of more than eight members; generally, various members are believed to have overlapping function in the cell [2]. HSP70 proteins play a role in the mediation of correct protein folding, and accordingly, maintains protein homeostasis [3]. Such proteins also boost cell survival following stresses, including higher temperature, hypoxia, oxidative stress, altered $\mathrm{pH}$, heavy metals etc. This survival role is revealed in the ability of HSP70 to decrease the toxicity of denatured and misfolded proteins that accumulate during stress. Cancer cells dependence for HSP70 is the rational for its targeting in cancer therapy. Much effort has been specified in the last years for the active search of HSP70 inhibitors [4].

Different types of human tumors overexpress different members of HSP70 family, and their expression generally are markers for poor cancer prognosis. Therefore, inhibitors of the HSP70 have appeared as important anticancer agents, intense focus has now been done on the potential for HSP70 inhibitors to assume a role as a significant chemotherapeutic agent. 
Rosmarinic acid and sinesitin are some known active compounds generally available in plants belonging to Lamiaceae family. These compounds have number of essential biological activities, e.g. antiviral, antibacterial, anti-inflammatory, anticancer and antioxidant [5].

Computational study like molecular docking is one of the best techniques to evaluate the inhibitory effects of rosmarinic acid and sinesitin for HSP70. This study helps to identify the optimum geometry of compound- receptor complex and estimate their energy interactions.

\section{METHODS}

\subsection{Docking method using AutoDock4.2}

Various language programs were downloaded from various sources; Python (www.python.com), Molecular graphics laboratory(MGL) tools (http://mgltools.scripps.edu) and AutoDock4.2 ( http://autodock.scripps.edu), Bio Via draw ( http://accelrys.com), Discovery studio visualizer 2017 ( http://accelrys.com) and Chem3D ( https://acms.ucsd.edu).

Computational study like molecular docking is one of the best techniques to evaluate the inhibitory effects of active compounds such as rosmarinic acid and sinesitin for HSP70.

Crystal structure of antiangiogenic target heat shock protein70 (HSP70) with PDB ID : 4HWI was selected and downloaded from Protein Data Bank (www.rvcsb.org/pdb) (Figure 1) [6].

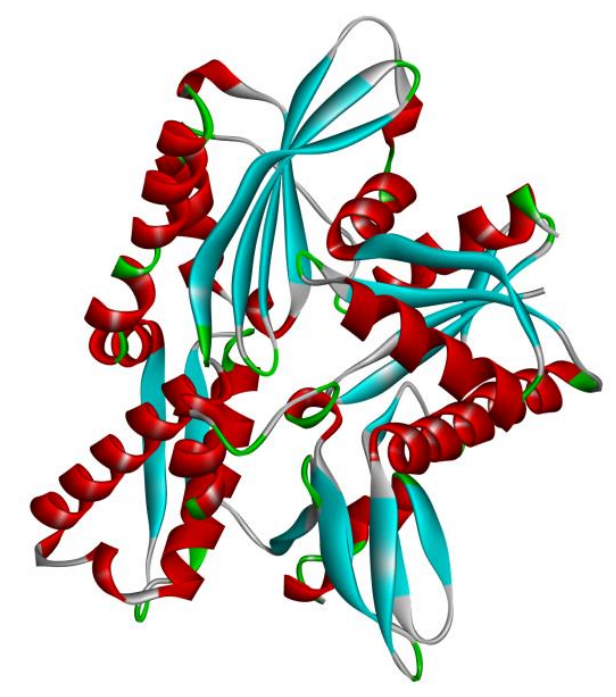

Figure 1: Heat shock protein from RCSB protein data bank (4HWI)

The starting structure of heat shock protein was prepared using AutoDock tools. Water molecule was deleted, polar hydrogen and Kollman charges were added to the heat shock protein starting structure. Grid box was set with the size of $78 \times 82 \times 50 \AA$ with the grid spacing of $0.375 \AA$ at the binding site. The starting structure for all the ligands namely rosmarinic acid and sinesitin, were constructed using Bio Via draw. Gasteiger charges were assigned into optimized ligand using AutodockTools. 100 docking runs were conducted with mutation rate of 0.02 and crossover rate of 0.8 . The population size was set to use 250 randomly placed individual. Lamarckian Genetic algorithm was used as the searching algorithm with a 
translational step of $0.2 \AA$, a quaternion step of $5 \AA$ and a torsion step of $5 \AA$. Most populated and lowest binding free energy

\section{RESULTS AND DISCUSSION}

The docked conformation of HSP70 with rosmarinic acid and sinesitin clearly revealed that numerous potential interactions were present. Rosmarinic acid has shown four conventional hydrogen bonds after docking with HSP70 molecule. The result demonstrated that it interacts with ASN364, ASP366, ARG272 and SER340. It caused strong conventional hydrogen bindings with average free binding energy of -6.28 $\mathrm{kcal} / \mathrm{mol}$.

Sinesitin (Figure 2) interacted with SER340 of HSP70 via conventional hydrogen binding It caused conventional hydrogen bindings with average free binding energy of $-5.83 \mathrm{kcal} / \mathrm{mol}$. The binding sites of ATP as standard (Figure 2) after interaction with HSP70 showed conventional hydrogen bonds with ARG342, ASP366, GLY202, GLY203, GLY230 and THR14. It showed six hydrogen bonds with the average free binding energy of $-3.95 \mathrm{kcal} / \mathrm{mol}$.

The minimum free binding energy of each ligand to protein HSP70 has been shown in table 1. It reveals that the affinity of the active compounds rosmarinic acid and sinesitin are almost in same range and significantly more than the affinity of the HSP70 towards its normal substrate, which considered free ATP in this study.

Table1. Free binding energy of the compounds

\begin{tabular}{|c|c|}
\hline Ligand & $\begin{array}{c}\text { Lowest Binding } \\
\text { energy }(\mathrm{kcal} / \mathrm{mol})\end{array}$ \\
\hline Rosmarinic acid & -6.28 \\
\hline Sinesitin & -5.83 \\
\hline ATP & -3.95 \\
\hline
\end{tabular}

Various studies were performed with the practical screening of different active compounds to inhibit HSP70. The need of valid practical data is a major challenge of compound development in the step of development in the purpose of accuracy in computational models. The docking values like hydrogen bond interactions, $\Pi-\Pi$ interactions, free binding energy and direction of docked ligand in the active site of protein have been obtained to evaluate the results. According to the in silico evaluation and stereochemistry binding of active compounds, hsp70 affinities towards rosmarinic acid and sinesitin are more compared to ATP which is its normal substrate. This affinity shows the competition, which is more possibility for these compounds to bind to the HSP70 rather than ATP. Even though ATP can interact using 6 hydrogen bonds but the average of free binding energy after interaction with rosmarinic acid and sinesitin is less than its interaction with HSP70. This happened probably because rosmarinic acid and sinesitin have one aromatic ring, which makes the interaction stable enough to happen [7]. However, rosmarinic acid has higher affinity than sinesitin to HSP70 due to lower binding energy, which is due to more hydron bonds after interaction with HSP70. Rosmarinic acid probably has more potential to bind to HSP70 compared with sinesitin. However, this needs more study to confirm it.

These results demonstrate that the potent binding sites are available in the selected active compounds compared with the standard substrate. It confirms the potential of mentioned active compounds to inhibit the HSP70 protein. 

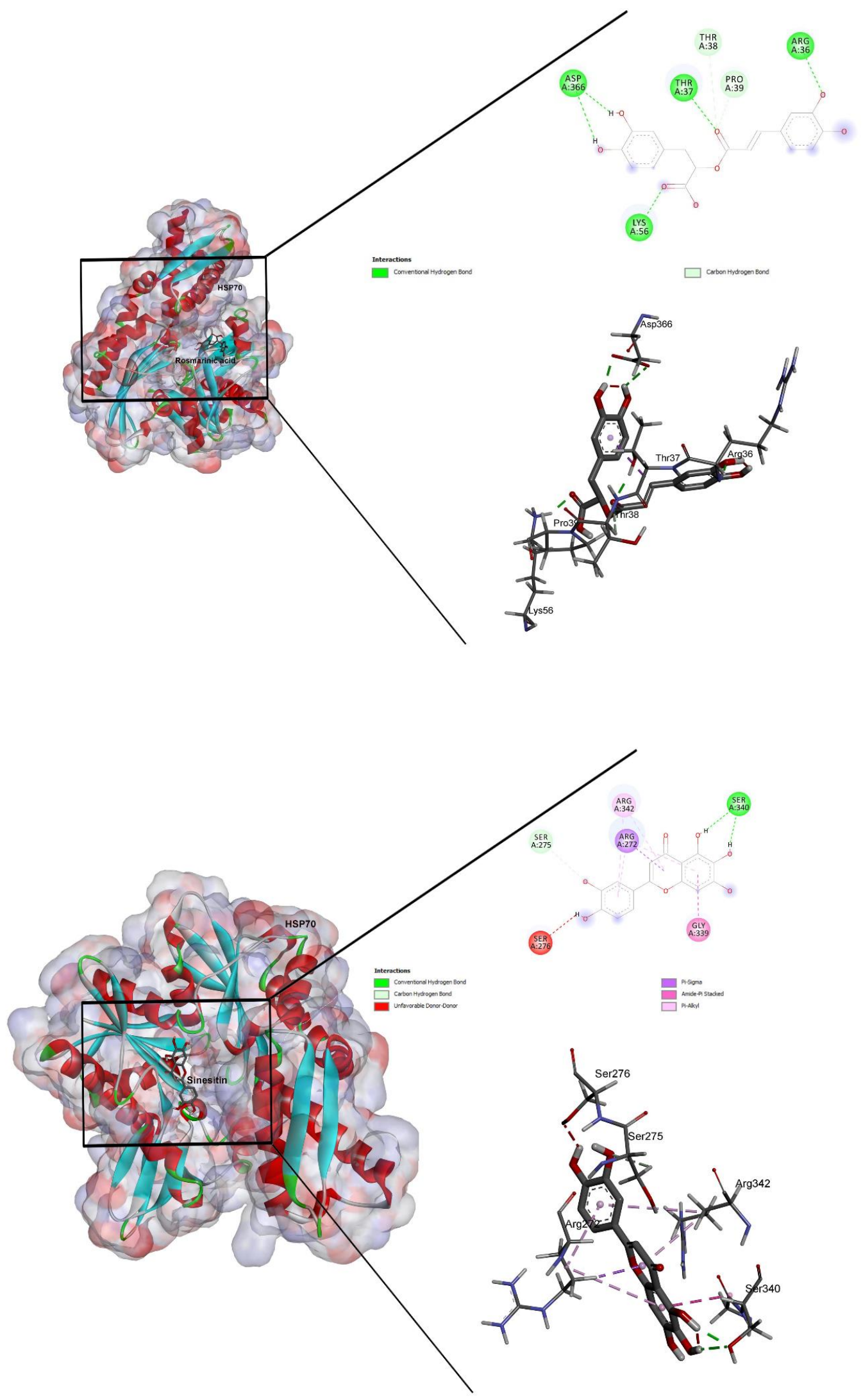


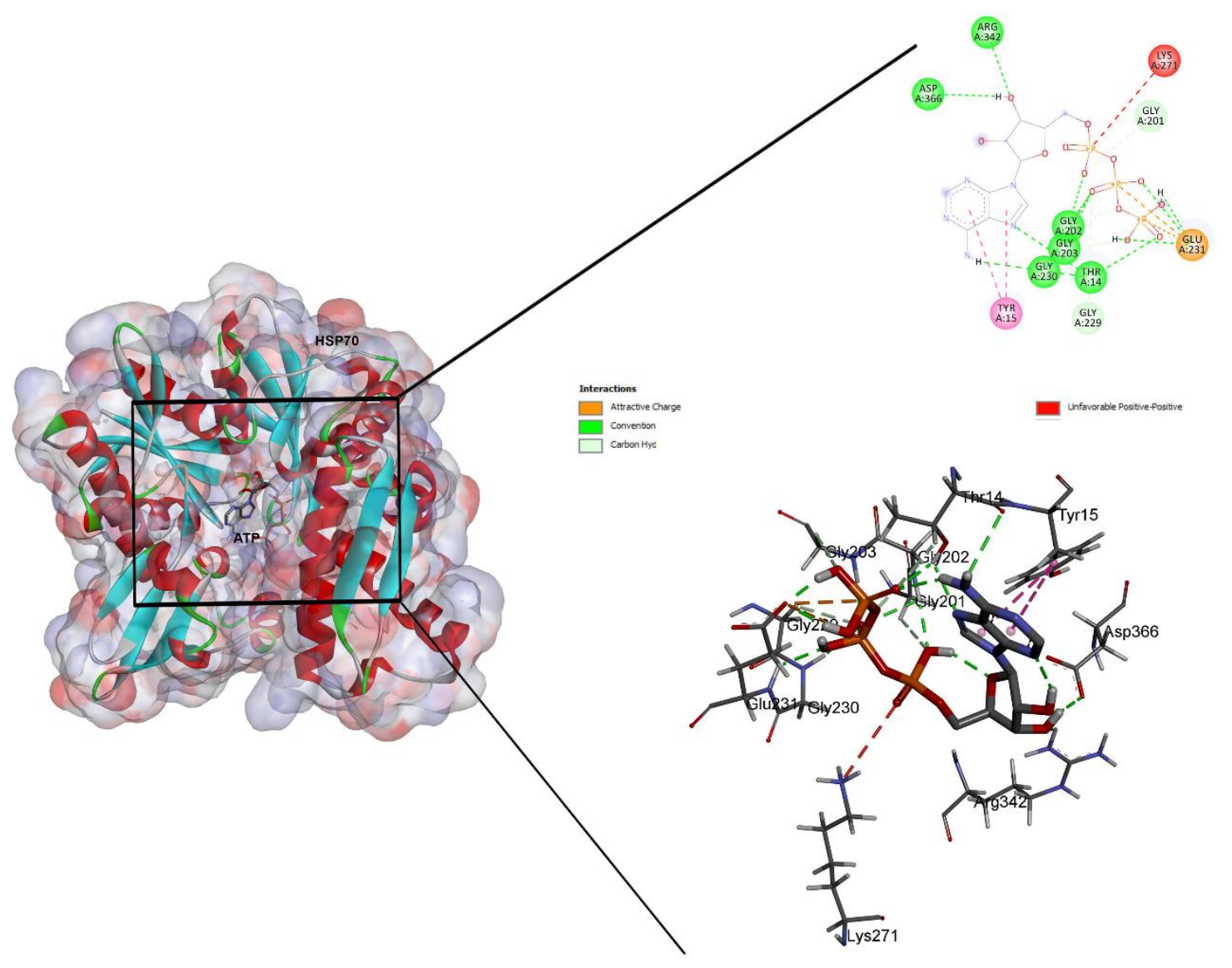

Figure 2: Docked posture of HSP70 protein with the selected ligands (rosmarinic acid, sinesitin and ATP)

\section{CONCLUSION}

In conclusion, the docking study revealed that the rosmarinic acid among all the other active compounds may have better binding site and more chances of interactions with HSP70 compare to standard. Based on this computational study the plant extract, which contains such active compounds, can have the potential to have anticancer effect.

\section{ACKNOWLEDGMENT}

This work was financially supported by a Research Grant (Project No. RDU 180336) from Universiti Malaysia Pahang (www.ump.edu.my), Malaysia, for which the authors are very grateful. 


\section{REFERENCES}

[1] Torre, L.A.;F. Bray;R.L. Siegel;J. Ferlay;J. Lortet-Tieulent, and A. Jemal, Global cancer statistics, 2012, CA: a cancer journal for clinicians, 2015, 65(2), 87-108.

[2] Tavaria, M.;T. Gabriele;I. Kola, and R.L. Anderson, A hitchhiker's guide to the human Hsp70 family, Cell stress \& chaperones, 1996, 1(1), 23.

[3] O Tiroli-Cepeda, A. and C. HI Ramos, An overview of the role of molecular chaperones in protein homeostasis, Protein and peptide letters, 2011, 18(2), 101-109.

[4] Goloudina, A.R.;O.N. Demidov, and C. Garrido, Inhibition of HSP70: a challenging anticancer strategy, Cancer letters, 2012, 325(2), 117-124.

[5] Vishkaei, M.N.;S.B.S.H. Sultan;M.B. Khadeer, and A.M. Ahamed, Phospholipid-encapsulated herbal extract to enhance anti-angiogenic activity: Phytosomes in angiotherapy, World Journal of Pharmaceutical Sciences, Regulation, 2017, 15, 7.

[6] Fang, S.;L. Li;B. Cui;S. Men;Y. Shen, and X. Yang, Structural insight into plant programmed cell death mediated by BAG proteins in Arabidopsis thaliana, Acta Crystallographica Section D: Biological Crystallography, 2013, 69(6), 934-945.

[7] Gottschalk, H.C.;J. Altnöder;M. Heger, and M.A. Suhm, Control over the Hydrogen-Bond Docking Site in Anisole by Ring Methylation, Angewandte Chemie International Edition, 2016, 55(5), 1921-1924. 